\title{
Nonlinear Frequencies for Transverse Free Oscillations of a Transporting Tensioned Beam
}

\author{
Hu Ding ${ }^{1, a}$, Liqun Chen ${ }^{2, a, b}$ and Haiyan Jiang ${ }^{3, c}$ \\ ${ }^{1}$ Shanghai Institute of Applied Mathematics and Mechanics, Shanghai Key Laboratory of \\ Mechanics in Energy Engineering, Shanghai University, Shanghai 200072, China \\ ${ }^{2}$ Department of Mechanics, Shanghai University, Shanghai 200444, China \\ ${ }^{3}$ School of Electronic Science \& Applied Physics, Hefei University of Technology, Hefei, Anhui, \\ 230009, China

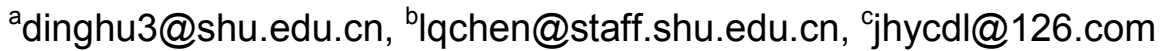

Keywords: Axially moving beams, Nonlinearity, oscillations, Natural frequency, Fast Fourier transform, The differential quadrature

\begin{abstract}
This study focuses on the natural frequencies of nonlinear free transverse oscillations of transporting tensioned beams via the standard fast Fourier transform (FFT). The transverse oscillations of transporting tensioned beams can be governed by a nonlinear partial-differential equation or a nonlinear integro-partial-differential equation. Numerical schemes are respectively presented for the two governing equations via the differential quadrature method. For each nonlinear equation, the nonlinear oscillations frequencies are investigated via FFT with the time responses histories. The numerical results depict the tendencies of the frequencies of nonlinear free transverse oscillations of transporting tensioned beams with the changing oscillations amplitude, transporting speed, the nonlinear coefficient and the flexural stiffness.
\end{abstract}

\section{Introduction}

The axially moving structures have received a great deal of attention due to their manifestation in a wide class of engineering fields. The belt drives, power transmission band, band saw blades, and high-speed magnetic tapes are the typical examples of such axially moving structures. Most of the one-dimensional structures with flexural rigidity, which are axially moving over two supports, have been represented by the beam models. Understanding transverse vibrations of axially moving beams is important for the design of the devices.

The wide diffusion of axially moving systems in industrial processes has motivated intense research activity. Mote [1] first investigated the first three frequency and modes for simple supported boundary conditions via the Galerkin method. Wickert and Mote [2] presented a complex model method for axially moving continua including beams where natural frequencies and modes associated with free vibration serve as a basis for analysis. Öz and Pakdemirli [3] and Öz [4] computed the first two natural frequencies values in the cases of pinned-pinned ends and clampedclamped ends, respectively. Kong and Parker [5] combined perturbation techniques for algebraic equations and phase closure principle to determine approximate natural frequencies of an axially moving beam with small flexural stiffness. Ding and Chen [6] gave the first two frequencies of axially moving elastic and viscoelastic beams on simple supports with torsion springs. Ghayesh and Khadem [7] calculated natural frequency for the first two modes in free non-linear transverse vibration of an axially moving beam in which rotary inertia and temperature variation effects have been considered. Matbuly et al. [8] employed the method of differential quadrature to determine the natural frequencies and the mode shapes for the free vibration of an elastically supported cracked beam. Özkaya et al. [9] investigated natural frequencies for a slightly curved beam carrying a concentrated mass. All of above literatures, the natural frequency of axially moving beams was calculated from linear governing equation of transverse vibration. The known exception is that Wickert [10] used a perturbation method to investigate the fundamental frequency of axially moving materials from a nonlinear integro-partial-differential equation of transverse vibration. 
However, so far there are very limited researches on the first few natural frequencies of nonlinear vibration. To address the lacks of research in this aspect, the present investigation studies the first two natural frequencies of nonlinear free transverse vibration of axially moving beams via the numerical solutions and the FFT.

The nonlinear integro-partial-differential equation and the nonlinear partial-differential equation both can govern the transverse motion of an axially moving beam. The nonlinear integropartial-differential equation has been used by Wickert [10], Pellicano and Vestroni [11], Parker and Lin [12], Suweken and van Horssen [13]. The nonlinear partial-differential equation has used by Öz et al. [14], Marynowski [15], Ghayesh and Khadem [7]. As summarized in [16, 17], both nonlinear models for transverse vibration of axially moving beams have been widely used. The two models were compared for parametric vibration [17] of axially moving beams. It is found that the predictions made by the two models are qualitatively the same, but quantitatively different. The transverse responses of the two models were compared with the transverse component of the coupled equation for free vibration $[16,18,19]$ and forced vibration [20] of axially moving beams. Both models yield almost the same precision results for some conditions and the integro-differential equation always gives better results. However, so far it has not been clear which model yields better outcomes. So the natural frequencies of axially moving beams are calculated for both models in the investigation.

The present paper is organized as follows. Section 2 establishes the coupled governing equation and two equations for transverse motion of an axially moving beam. Section 3 presents develops the finite difference schemes to solve the coupled equations of planar motion presented in Section 2. Section 4 presents develops the fast Fourier transform schemes to solve the natural frequency from the numerical results presented in Section 3. Section 5 compares the coupled equations of planar motion with two governing equations of transverse motion via the natural frequency. Section 6 ends the paper with the concluding remarks.

\section{Mathematical models}

Consider a uniform beam is moving in its axial direction at a uniform constant transport speed of $\gamma$ between two boundaries. The span between two boundaries is $l$. The structural properties of the beam are given by the mass density $\rho$, the cross-sectional area $A$, the moment of inertial $I$, the initial tension $P_{0}$, and the Young's modulus $E$. Assume the beam has small amplitude vibrations in the axial and transverse directions. For a slender beam, the linear moment-curvature relationship is sufficiently accurate. The fixed axial coordinate $x$ measure the distance from the left boundary. The transverse displacement $v(x, t)$ related to a spatial frame. The beam is subjected to no external loads. The nonlinear the partial-differential equation and the integro-partial-differential equation for transverse motion of axially moving elastic beam can be cast into the dimensionless form [16]

$$
v_{,_{t t}}+2 \gamma v,_{x t}+\left(\gamma^{2}-1\right) v,_{x x}+k_{\mathrm{f}}{ }^{2} v_{x x x x}=\frac{3}{2} k_{1}^{2} v_{,_{x}}{ }^{2} v,_{x x}
$$

and

$$
v_{,_{t t}}+2 \gamma,_{x t}+\left(\gamma^{2}-1\right) v,_{x x}+k_{\mathrm{f}}^{2} v_{x x x x}=\frac{1}{2} k_{1}^{2} v_{,_{x x}} \int_{0}^{1} v_{x_{x}}^{2} \mathrm{~d} x
$$

where a comma preceding $x$ or $t$ denotes partial differentiation with respect to $x$ or $t$, and The dimensionless variables and parameters as follows

$$
v \leftrightarrow \frac{v}{l}, x \leftrightarrow \frac{x}{l}, t \leftrightarrow t \sqrt{\frac{P_{0}}{\rho A l^{2}}}, \gamma \leftrightarrow \gamma \sqrt{\frac{\rho A}{P_{0}}}, k_{1}=\sqrt{\frac{E A}{P_{0}}}, k_{\mathrm{f}}=\sqrt{\frac{E I}{P_{0} l^{2}}}
$$

In the present investigation, only the boundary conditions of the beam is fixed at both ends are considered as follows 


$$
v(0, t)=v(1, t)=0, v,_{x}(0, t)=v,_{x}(1, t)=0
$$

In this paper, the natural frequencies of nonlinear transverse vibration of an axially moving beam are focused on.

\section{Numerical methods}

In the following investigation, the differential quadrature method [16] is applied to calculate time responses of transverse vibration from equations (1) and (2) under fixed boundary conditions (4). Introduce $N$ unequally spaced sampling points as

$$
\begin{aligned}
& x_{i}=\frac{1}{2}\left[1-\cos \frac{(i-1) \pi}{N-3}\right], \quad(i=3,4, \ldots, N-2), \\
& x_{1}=0, x_{2}=0.0001, x_{N-1}=1-0.0001, x_{N}=1
\end{aligned}
$$

The quadrature rules for the derivatives of a function at the sampling points yield

$$
v_{x_{x}}\left(x_{i}, t\right)=\sum_{j=1}^{N} A_{i j}^{(1)} v\left(x_{j}, t\right), v,_{x x}\left(x_{i}, t\right)=\sum_{j=1}^{N} A_{i j}^{(2)} v\left(x_{j}, t\right), v,_{x x x x}\left(x_{i}, t\right)=\sum_{j=1}^{N} A_{i j}^{(4)} v\left(x_{j}, t\right)
$$

where the weighting coefficients are as follows

$$
A_{i j}^{(1)}=\frac{\prod_{k=1, k \neq i}^{N}\left(x_{i}-x_{k}\right)}{\left(x_{i}-x_{j}\right) \prod_{k=1, k \neq j}^{N}\left(x_{j}-x_{k}\right)}(i, j=1,2, \cdots, N ; j \neq i)
$$

and the recurrence relationship

$$
\begin{aligned}
& A_{i j}^{(r)}=r\left[A_{i i}^{(r-1)} A_{i j}^{(1)}-\frac{A_{i j}^{(r-1)}}{x_{i}-x_{j}}\right](r=2,3,4,5 ; i, j=1,2, \cdots, N ; j \neq i) \\
& A_{i i}^{(r)}=-\sum_{k=1, k \neq i}^{N} A_{i k}^{(r)}(r=1,2,3,4,5 ; i=1,2, \cdots, N)
\end{aligned}
$$

The weighting coefficients $I_{g}(g=1,2, \ldots, N)$ for integrals are solved from [16]

$$
\left(\begin{array}{ccccc}
1 & 1 & \cdots & 1 & 1 \\
x_{1} & x_{2} & \cdots & x_{N-1} & x_{N} \\
\vdots & \vdots & & \vdots & \vdots \\
x_{1}{ }^{N-2} & x_{2}{ }^{N-2} & \cdots & x_{N-1}{ }^{N-2} & x_{N}{ }^{N-2} \\
x_{1}{ }^{N-1} & x_{1}{ }^{N-1} & \cdots & x_{N-1}{ }^{N-1} & x_{N}{ }^{N-1}
\end{array}\right)\left(\begin{array}{c}
I_{1} \\
I_{2} \\
\vdots \\
I_{N-1} \\
I_{N}
\end{array}\right)=\left(\begin{array}{c}
1 \\
1 / 2 \\
\vdots \\
1 /(N-1) \\
1 / N
\end{array}\right)
$$

Discretization of the spatial variables in Eqs. (1) and (2) via substituting Eq. (6) and $I_{g}$ into Eqs. (1) and (2) yields

$$
\begin{aligned}
& \ddot{v}_{i}+\left[\sum_{j=1}^{N} 2 \gamma A_{i j}^{(1)}\right] \dot{v}_{i}-\sum_{j=1}^{N}\left[\left(\gamma^{2}-1\right) A_{i j}^{(2)}+k_{\mathrm{f}}^{2} A_{i j}^{(4)}\right] v_{j}=\frac{3}{2} k_{1}^{2}\left(\sum_{j=1}^{N} A_{i j}{ }^{(2)} v_{j}\right)\left(\sum_{j=1}^{N} A_{i j}{ }^{(1)} v_{j}\right)^{2}, \\
& (i=3,4, \ldots, N-2) \text {, } \\
& v_{1}=v_{N}=\sum_{j=1}^{N} A_{2 j}{ }^{(1)} v_{j}=\sum_{j=1}^{N} A_{(N-1) j}{ }^{(1)} v_{j}=0
\end{aligned}
$$


and

$$
\begin{aligned}
& \ddot{v}_{j}+\dot{v}_{k} \sum_{k=1}^{N} 2 \gamma A_{j k}{ }^{(1)}+\sum_{k=1}^{N}\left[\left(\gamma^{2}-1\right) A_{j k}{ }^{(2)}+k_{\mathrm{f}}{ }^{2} A_{j k}{ }^{(4)}\right] v_{k}=\frac{1}{2} k_{1}^{2}\left(\sum_{k=1}^{N} A_{j k}{ }^{(2)} v_{k}\right) \sum_{g=1}^{N} I_{g}\left(\sum_{k=1}^{N} A_{g k}{ }^{(1)} v_{k}\right)^{2} \\
& (j=3,4, \ldots, N-2) \\
& v_{1}=v_{N}=\sum_{j=1}^{N} A_{2 j}{ }^{(1)} v_{j}=\sum_{j=1}^{N} A_{(N-1) j}{ }^{(1)} v_{j}=0
\end{aligned}
$$

Substitution of boundary condition (4) makes $v\left(x_{i}, t\right)$ solvable for a set of given parameters $k_{\mathrm{f}}, \gamma, k_{1}$, initial conditions. In this way, equation (1) or (2) can be solved numerically. For an odd $N$, $v\left(x_{(N+1) / 2}, t\right)$ is the beam center displacement.

\section{The oscillation frequencies of nonlinear models}

In all numerical examples here, the initial conditions are chosen as second eigenfunction of a stationary beam under the fixed boundary conditions, namely

$$
v(x, 0)=D\left\{\cosh \left(\beta_{1} x\right)-\cos \left(\beta_{1} x\right)+\zeta_{1}\left[\sin \left(\beta_{1} x\right)-\sinh \left(\beta_{1} x\right)\right]\right\}, v,,_{x}(x, 0)=0
$$

where

$$
\zeta_{1}=\frac{\cosh \beta_{1}-\cos \beta_{1}}{\sinh \beta_{1}-\sin \beta_{1}}, \beta_{1}=7.8532
$$

and $D$ represents the amplitude of vibration. The transverse displacement of the beam centre for equations (1) and (2) will be numerically solved via the differential quadrature schemes under the boundary conditions (4) and the initial conditions (13).

The Fourier transform has been widely used in circuit analysis and synthesis, from filter design to signal processing and image reconstruction. The principle of transform in engineering is to find a different representation of a signal under investigation. The discrete Fourier transform (DFT) is an approximation of the Fourier transform in a digital environment for computing the Fourier transform numerically on a computer. The FFT is an algorithm to speed up DFT computation. The FFT forces one further assumption: that $N$ is an integer multiple of two. A good tutorial on the FFT can be found in [21]. The maximum frequency in the FFT depends on the sampling interval, and the frequency resolution is determined by the record length of the signal. That is, $N$ samples of a time signal recorded during a finite duration of $T$ with a sampling period of $\Delta t(N=T / \Delta t)$ can be transformed into $N$ samples in the frequency domain. In the present investigation, the natural frequencies of the nonlinear transverse vibration of axially moving beams are calculated from the time signals of the transverse center displacement the of beam via the FFT, and $N=4096, \Delta t=0.025$.

Consider a beam with modulus of elasticity $E=2.1 \times 10^{11} \mathrm{~Pa}$ and density $\rho=7850 \mathrm{~kg} / \mathrm{m}^{3}$. Let the initial tension $P_{0}=7850 \mathrm{~N}$, the axial speed $\gamma=51.72 \mathrm{~m} / \mathrm{s}$, and the cross-section of the beam being a rectangle with the width $W=0.0135 \mathrm{~m}$ and the height $H=0.0277 \mathrm{~m}$. Then equation (3) yields $\gamma=1.0$ , $k_{\mathrm{f}}=0.8, k_{1}=100$. As $k_{1}$ represents the effect of nonlinearity, it is called the nonlinear coefficient. Based on the numerical solutions of equation (1) and (2) under the fixed boundary condition, Figs. 1 and 3 respectively show the transverse displacement of the beam center for numerical solution of Eq. (1) and Eq. (2). In Figs. 1 and 3, the vibration amplitude $D=0.0001$. Figs. 2 and 4 respectively illustrate the results of the first three natural frequencies of the beam which calculated via the FFT from the Fig. 1 and Fig.3. In Figs.2 and 4, $\omega$ represents the frequencies. 




(a) The time history



(b) Zoom view

Fig. 1. The transverse motion of the midpoint of beam: Eq. (1)



Fig. 2. The power spectrum of Fig.1

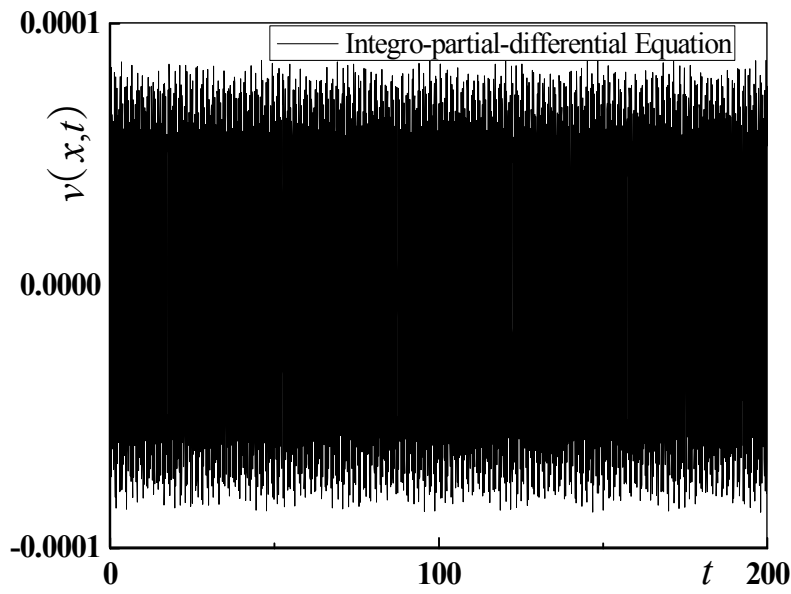

(a) The time history



Fig. 4. The power spectrum of Fig. 3.



(b) Zoom view

Fig. 3. The transverse motion of the midpoint of beam: Eq. (2)

Figs. 5 and 6 respectively illustrate the effects of the nonlinear coefficient with $k_{\mathrm{f}}=0.8$ and the vibration amplitude $D=0.0001$ on the first two natural frequencies of Eq. (1) and Eq. (2) versus axial speed. In Figs.5 and 6, the dash-dot lines, the solid lines and the dots respectively stand for the natural frequencies to $k_{1}=2000, k_{1}=100$ and $k_{1}=0$. The nonlinear coefficient $k_{1}=0$, means the natural 
frequencies are calculated from the linear elastic system. For the given $k_{1}$, the natural frequencies decrease with the growth of axial speed. The comparisons also indicate that the nonlinear coefficient $k_{1}$ has little effects on the natural frequency when vibration is rather small, especially for the small axial speed and the first natural frequency, even if the nonlinear coefficient rather large.



(a)The first natural frequency



(b) The second natural frequency

Fig. 5. The effects of the nonlinear coefficient on the natural frequencies versus axial speed: Eq. (1)



(a)The first natural frequency

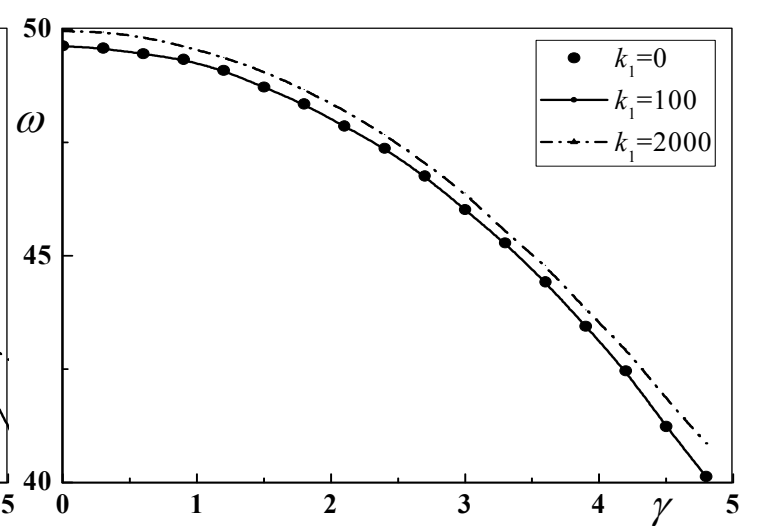

(b) The second natural frequency

Fig. 6. The effects of the nonlinear coefficient on the natural frequencies versus axial speed: Eq. (2)

Figs. 7 and 8 respectively illustrate the effects of the nonlinear coefficient with $k_{\mathrm{f}}=0.8$ on the first two natural frequencies of Eq. (1) and Eq. (2) versus vibration amplitude. In Figs.7 and 8, the dash-dot lines, the solid lines and the dots respectively stand for the natural frequencies to $k_{1}=150$, $k_{1}=100$ and $k_{1}=50$. For the given $k_{1}$, the natural frequencies increase with the growth of vibration amplitude. The comparisons also indicate that the first two natural frequencies increase with the nonlinear coefficient for the large vibration.

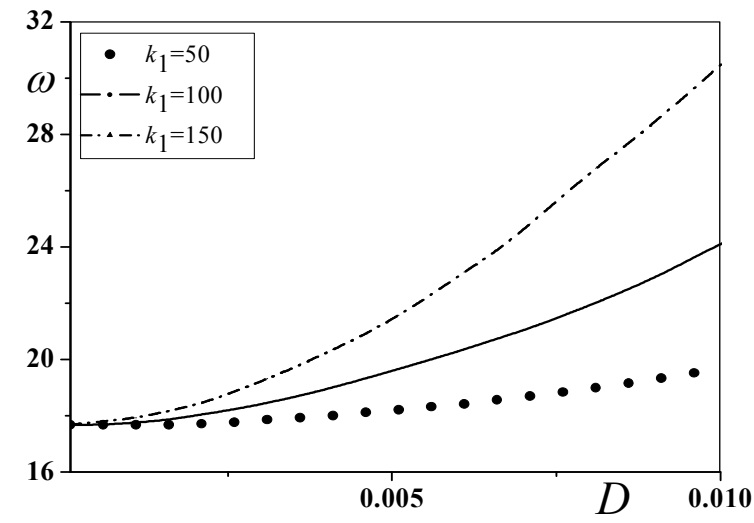

(a)The first natural frequency

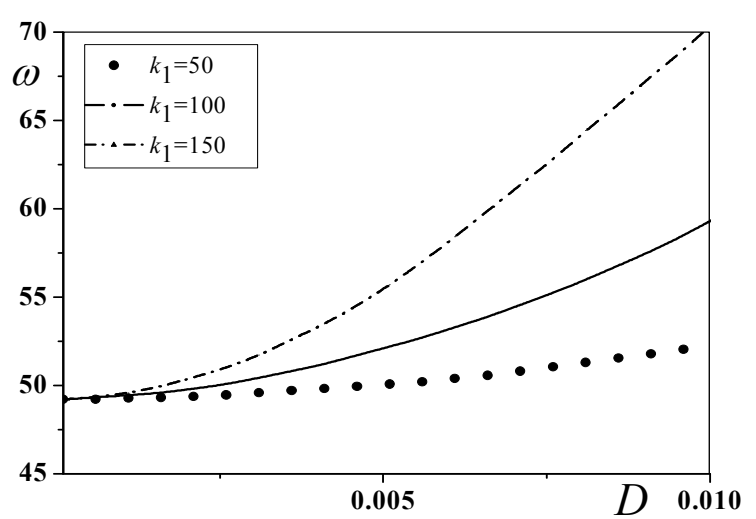

(b) The second natural frequency

Fig. 7. The effects of the nonlinear coefficient on the natural frequencies versus vibration amplitude:

Eq. (1) 


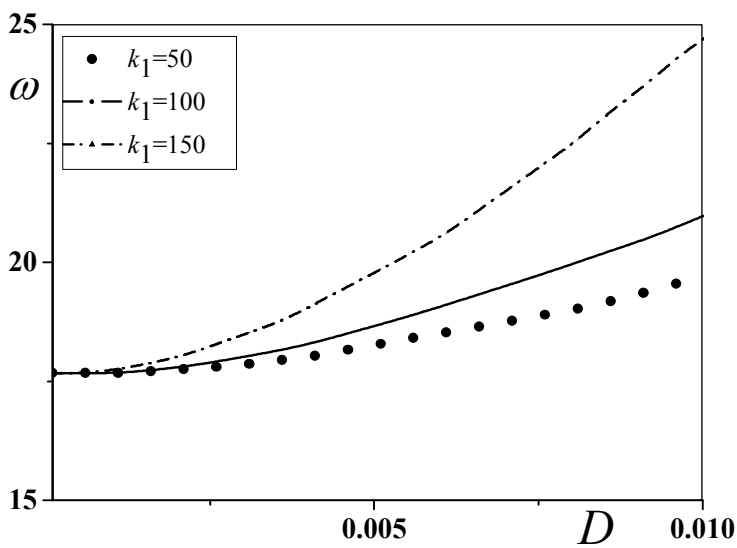

(a)The first natural frequency

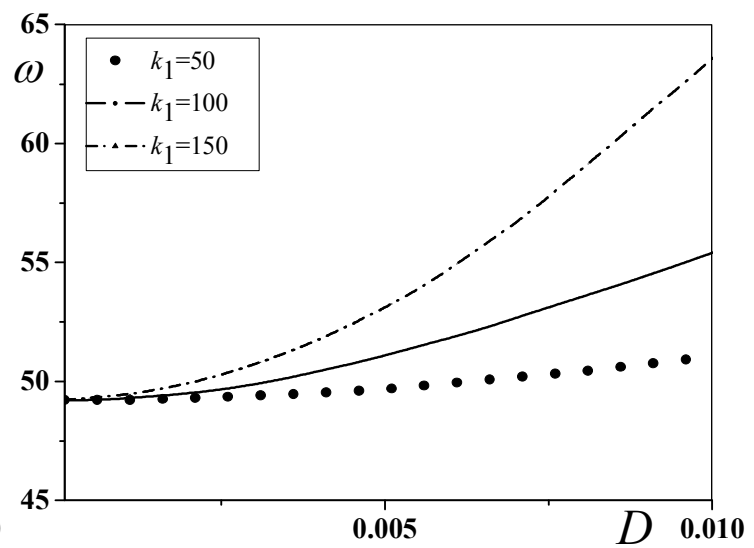

(b) The second natural frequency

Fig. 8. The effects of the nonlinear coefficient on the natural frequencies versus vibration amplitude: Eq. (2)

Figs. 9 and 10 respectively show the effects of the flexural stiffness with $k_{1}=100$ and $D=0.0001$ on the first two natural frequencies of Eq. (1) and Eq. (2). In Figs.9 and 10, the dash-dot lines, the dots and the solid lines respectively stand for the natural frequencies to $k_{\mathrm{f}}=1.0, k_{\mathrm{f}}=0.8$ and $k_{\mathrm{f}}=0.6$. For the given $k_{\mathrm{f}}$, the first two natural frequencies decrease with the growth of axial speed. The comparisons also indicate that the first two natural frequencies increase with the growth of the flexural stiffness $k_{\mathrm{f}}$.



(a)The first natural frequency

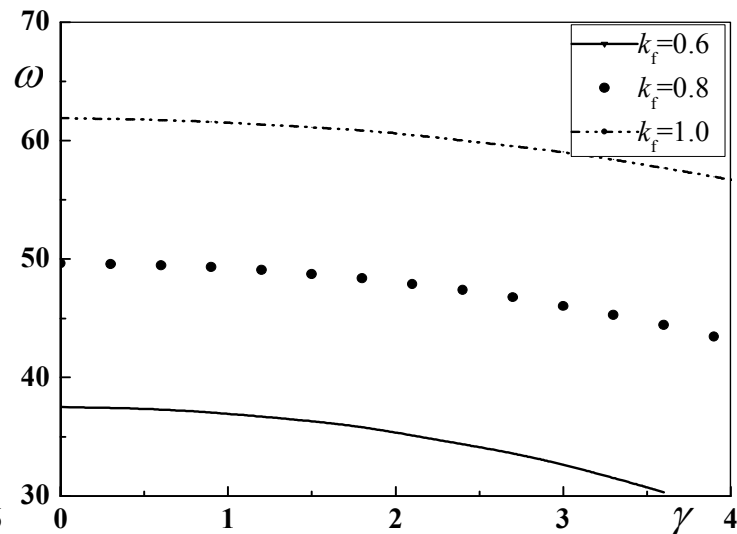

(b) The second natural frequency

Fig. 9. The effects of the flexural stiffness on the natural frequencies versus axial speed: Eq. (1)

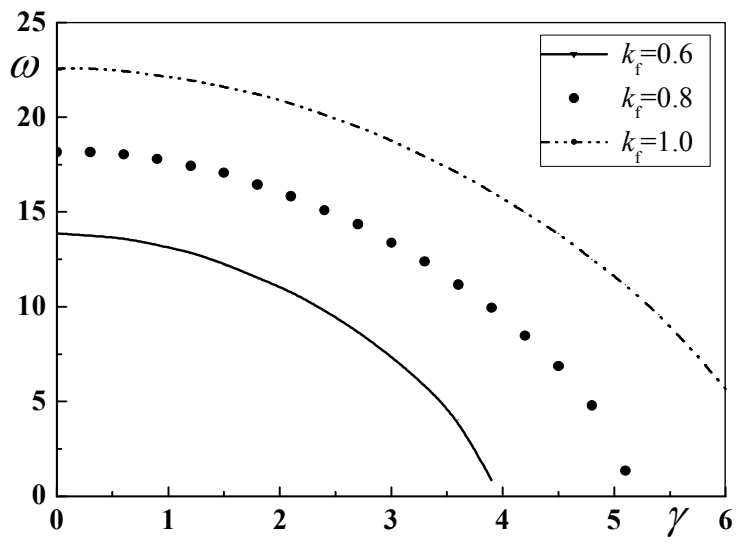

(a)The first natural frequency



(b) The second natural frequency

Fig. 10. The effects of the flexural stiffness on the natural frequencies versus axial speed: Eq. (2) 


\section{Comparisons}

Based on numerically solutions of the (1) and (2), the differences between the two nonlinear models can be investigated via the first two natural frequencies of equations by the FFT.

Fig.11 illustrates the natural frequencies versus axial speed with fixed the flexural stiffness $k_{\mathrm{f}}=0.6, k_{\mathrm{f}}=0.8$ and $k_{\mathrm{f}}=1.0$ respectively and the nonlinear coefficient $k_{1}=100$, the vibration amplitude $D=0.0001$. Fig. 11 shows that the natural frequencies of two models are overlapped with the changing axial speed $\gamma$ and flexural stiffness $k_{\mathrm{f}}$ for rather small vibration. The numerical results demonstrate that the flexural stiffness the axial speed has little effects on the different of the natural frequency of the two nonlinear models when the vibration is rather small. Figs.12 and 13 respectively illustrate the natural frequencies versus nonlinear coefficient and vibration amplitude with fixed the flexural stiffness $k_{\mathrm{f}}=0.8$ and the axial speed $\gamma=1.0$. In Fig. 12, the vibration amplitude $D=0.0001$. In Fig.13, the nonlinear coefficient $k_{1}=100$. The comparisons indicate that the natural frequencies of two models qualitatively predict the same tendencies with the changing nonlinear coefficient and vibration amplitude, while quantitatively, there are certain differences for really big nonlinear coefficient and rather large vibration, the difference increase with the nonlinear coefficient and vibration amplitude, and the natural frequencies from equation (2) are smaller to those from equation (1).

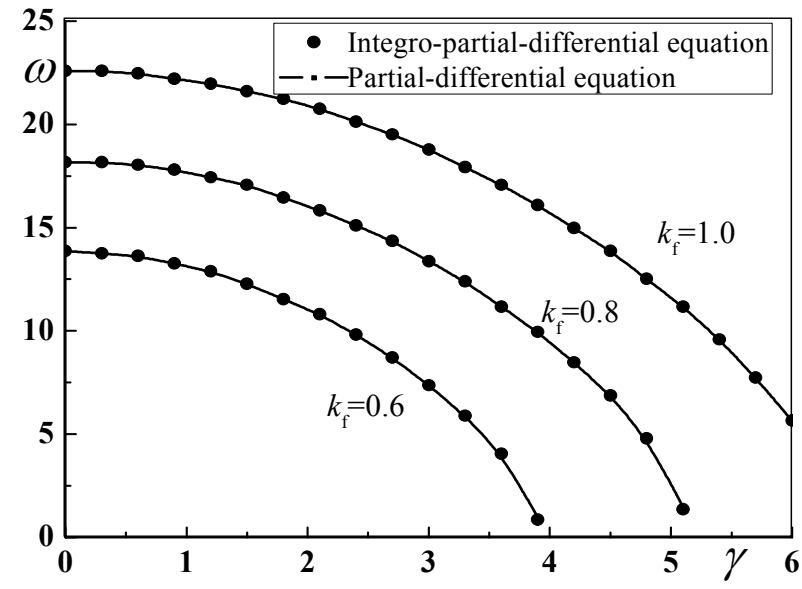

(a)The first natural frequency



(b) The second natural frequency

Fig. 11. The natural frequency calculated from equations (1) and (2) versus axial speed



(a)The first natural frequency

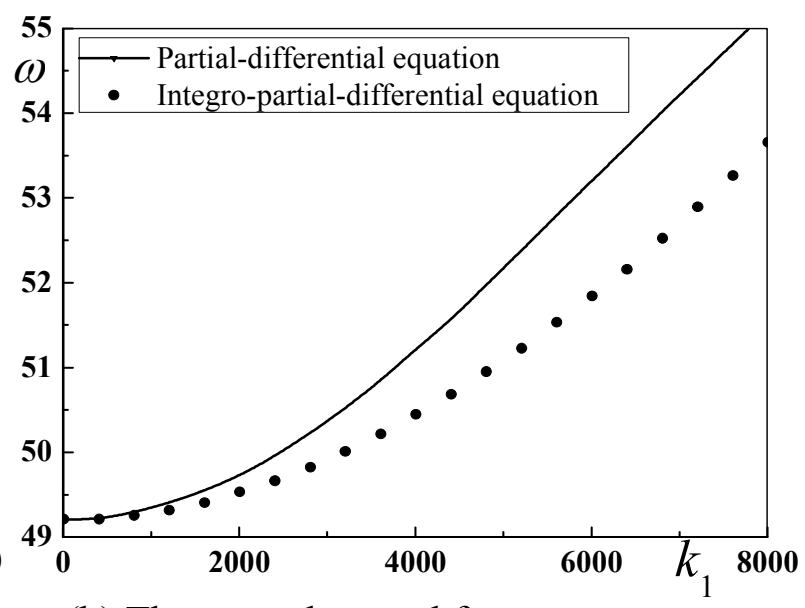

(b) The second natural frequency

Fig. 12. The natural frequency calculated from equations (1) and (2) versus nonlinear coefficient 


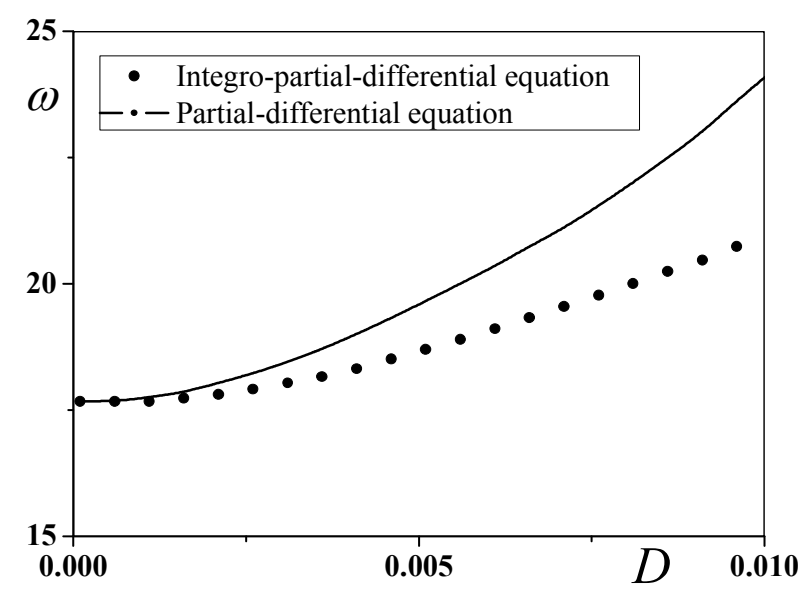

(a)The first natural frequency

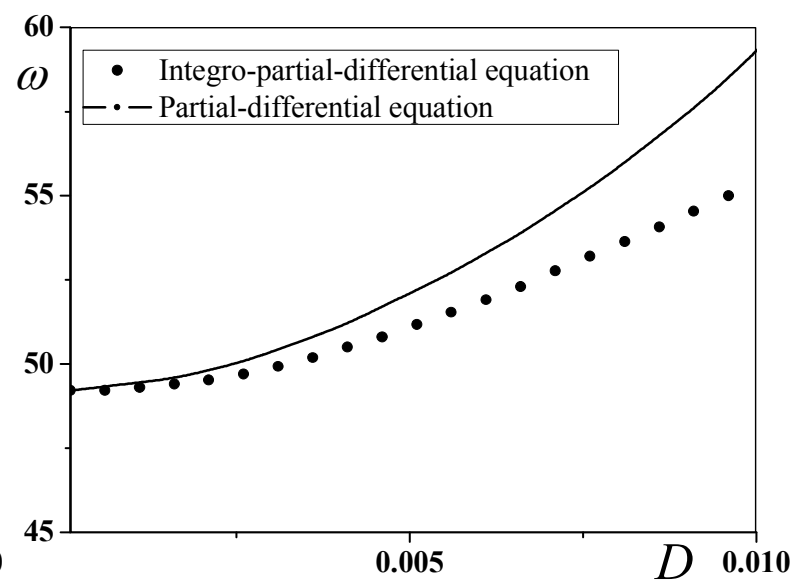

(b) The second natural frequency

Fig. 13. The natural frequency calculated from equations (1) and (2) versus vibration amplitude

\section{Conclusions}

Natural frequencies of nonlinear free oscillations of axially moving elastic beams are numerically investigated via the differential quadrature method and the fast Fourier transform. The transverse motion of an axially moving beam can be governed by a nonlinear partial-differential equation or a nonlinear integro-partial-differential equation. The time histories of transverse displacements of the centre of axially moving beams are respectively solved via the differential quadrature scheme for the two nonlinear models under the fixed boundary. The FFT is a computational tool for efficiently calculating the discrete Fourier transform of a series of data samples by means of digital computers. Time series of the discrete Fourier transform is defined as numerically solutions of two nonlinear governing equations. The standard fast Fourier transform is used to investigate the natural frequencies of nonlinear free transverse vibration of axially moving beams. The investigation leads to the following conclusions: (1) The nonlinear coefficient has little effects on the first two natural frequencies of nonlinear vibration of axially moving beams for rather small vibration, and the first two natural frequencies increase with the nonlinear coefficient for the large vibration. (2) The first two natural frequencies increase with the flexural stiffness and the vibration amplitude and decrease with the axially speed. (3) The two nonlinear models predict the same tendencies of the first two natural frequencies with the changing flexural stiffness, axially speed, nonlinear coefficient and vibration amplitude. (4) The nonlinear partial-differential equation leads to the bigger natural frequency for big nonlinear coefficient and rather large vibration and this difference increase with nonlinear coefficient.

\section{Acknowledgments}

This work was supported by the National Outstanding Young Scientists Fund of China (Project No. 10725209), the National Science Foundation of China (Project No. 10902064), Shanghai Subject Chief Scientist Project (No. 09XD1401700), Shanghai Leading Academic Discipline Project (No. S30106), and Shanghai Rising-Star Program (No. 11QA1402300).

\section{References}

[1] C.D. Mote Jr., Dynamic stability of an axially moving band, Journal of the Franklin Institute. 285 (1968) 329-346.

[2] J.A. Wickert, C.D. Mote Jr., Classical vibration analysis of axially moving continua, ASME Journal of Applied Mechanics. 57 (1990) 738-44.

[3] H.R. Öz and M. Pakdemirli, Vibrations of an axially moving beam with time dependent velocity, Journal of Sound and Vibration. 227 (1999) 239-257. 
[4] H.R. Öz, On the vibrations of an axially traveling beam on fixed supports with variable velocity, Journal of Sound and Vibration. 239 (2001) 556-564.

[5] L. Kong, R.G. Parker, Approximate eigensolutions of axially moving beams with small flexural stiffness, Journal of Sound and Vibration. 276 (2004) 459-469.

[6] H. Ding, L.Q. Chen, Stability of axially accelerating viscoelastic beams multi-scale analysis with numerical confirmations, European Journal of Mechanics A/Solids. 27 (2008) 1108-1120.

[7] M.H. Ghayesh, S.E. Khadem, Rotary inertia and temperature effects on non-linear vibration, steady-state response and stability of an axially moving beam with time-dependent velocity, International Journal of Mechanical Sciences. 50 (2008) 389-404.

[8] M.S. Matbuly, O. Ragb, M. Nassar, Natural frequencies of a functionally graded cracked beam using the differential quadrature method, Applied Mathematics and Computation. 215 (2009) 2307-2316.

[9] E. Özkaya, M. Sarigul, H. Boyaci, Nonlinear transverse vibrations of a slightly curved beam carrying a concentrated mass, Acta Mechanica Sinica. 25 (2009) 871-882.

[10] J.A. Wickert, Non-linear vibration of a traveling tensioned beam, International Journal of Non-Linear Mechanics. 27 (1992) 503-517.

[11] F. Pellicano, F. Vestroni, Nonlinear dynamics and bifurcations of an axially moving beam, ASME Journal of Vibration Acoustic 122 (2000) 21-30.

[12] R.G. Parker, Y. Lin, Parametric instability of axially moving media subjected to multifrequency tension and speed fluctuations, ASME Journal of Applied Mechanics. 68 (2001) 49-57

[13] G. Suweken and W.T. Van Horssen, On the weakly nonlinear, transversal vibrations of a conveyor belt with a low and time-varying velocity, Nonlinear Dynamics 31 (2003) 197-223

[14] H.R. Öz, M. Pakdemirli, H. Boyaci, Non-linear vibrations and stability of an axially moving beam with time-dependent velocity, International Journal Non-Linear Mechanics. 36 (2001) 107-115

[15] K. Marynowski, Non-linear vibrations of an axially moving viscoelastic web with timedependent tension, Chaos, Solitons and Fractals. 21(2004) 481-490.

[16] H. Ding, L.Q. Chen, On two transverse nonlinear models of axially moving beams, Science in China E. 52 (2009) 743-751.

[17] L.Q. Chen, H. Ding, Steady-state responses of axially accelerating viscoelastic beams: approximate analysis and numerical confirmation, Science in China G. 51 (2008) 1707-1721.

[18] H. Ding, L.Q. Chen, Galerkin methods for natural frequencies of high-speed axially moving beams, Journal of Sound and Vibration. 329 (2010) 3484-3494.

[19] H. Ding, L.Q. Chen, Natural frequencies of nonlinear vibration of axially moving beams, Nonlinear Dynamics. 63 (2011) 125-134

[20] H. Ding, L.Q. Chen, Nonlinear models for transverse forced vibration of axially moving viscoelastic beams, Shock and Vibration. 18 (2011) 281-287.

[21] P. Duhamel, M. Vetterli, Fast Fourier transforms: a tutorial review and a state of the art, Signal Processing. 19 (1990) 259-299 